\title{
Lattice points in Minkowski sums
}

\author{
Christian Haase, Benjamin Nill, Andreas Paffenholz * \\ Institut für Mathematik, Arnimallee 3, 14195 Berlin, Germany \\ \{christian.haase, nill, paffenholz\}@math.fu-berlin.de \\ Francisco Santos ${ }^{\dagger}$ \\ Facultad de Ciencias, Universidad de Cantabria, Av. de los Castros s/n, \\ E-39005 Santander, Spain \\ santosf@unican.es
}

Submitted: Dec 19, 2007; Accepted: Apr 1, 2008; Published: Apr 10, 2008

Mathematics Subject Classification: 52B20; 14M25

\begin{abstract}
Fakhruddin has proved that for two lattice polygons $P$ and $Q$ any lattice point in their Minkowski sum can be written as a sum of a lattice point in $P$ and one in $Q$, provided $P$ is smooth and the normal fan of $P$ is a subdivision of the normal fan of $Q$.

We give a shorter combinatorial proof of this fact that does not need the smoothness assumption on $P$.
\end{abstract}

\section{Introduction}

It is one of those problems. Everyone can understand it immediately. Yet, to this day we do not have any satisfactory solution.

A lattice polygon $P \subset \mathbb{R}^{2}$ is the convex hull of finitely many points in the lattice $\mathbb{Z}^{2}$. Given two lattice polygons $P$ and $Q$, we consider the addition map

$$
\begin{aligned}
\mathfrak{s}:\left(P \cap \mathbb{Z}^{2}\right) \times\left(Q \cap \mathbb{Z}^{2}\right) & \longrightarrow(P+Q) \cap \mathbb{Z}^{2} . \\
(x, y) & \longmapsto x+y
\end{aligned}
$$

We want to understand when $\mathfrak{s}$ is surjective. Equivalently, when is

$$
\left(P \cap \mathbb{Z}^{2}\right)+\left(Q \cap \mathbb{Z}^{2}\right)=(P+Q) \cap \mathbb{Z}^{2} ?
$$

\footnotetext{
${ }^{*}$ The first three authors were supported by Emmy Noether fellowship HA 4383/1 of the German research society DFG.

${ }^{\dagger}$ The fourth author was supported by grant MTM2005-08618-C02-02 of the Spanish Ministry of Science.
} 
This very basic question in discrete geometry (and its higher dimensional analogue) appears in different guises in algebraic geometry, commutative algebra, and integer programming. Specific cases also arise in additive number theory, representation theory, and statistics. Motivation for a conjectured sufficient condition comes from algebraic geometry.

Conjecture (Oda). Let $X$ be a smooth projective toric variety, let $D$ be an ample divisor on $X$, and let $D^{\prime}$ be nef. Then, the following homomorphism is surjective:

$$
H^{0}(X, \mathcal{O}(D)) \otimes H^{0}\left(X, \mathcal{O}\left(D^{\prime}\right)\right) \rightarrow H^{0}\left(X, \mathcal{O}\left(D+D^{\prime}\right)\right) .
$$

The toric dictionary translates this into discrete geometry as follows:

Conjecture (Oda'). Let $P$ and $Q$ be lattice polytopes. If $P$ is smooth and the normal fan of $Q$ coarsens that of $P$, then the map $\mathfrak{s}$ is surjective.

Here, a lattice polytope $P$ is called smooth if it is simple and at every vertex the primitive facet normals generate the dual lattice. This condition is equivalent to $P$ corresponding to an ample divisor on a smooth toric variety. The case $Q=n P, n \in \mathbb{N}$ of this conjecture is the conjecture that all smooth lattice polytopes are projectively normal.

The two-dimensional case of Oda's conjecture is now Fakhruddin's Theorem [Fak02], with an independent proof by Ogata Oga06. In this note, we generalize Fakhruddin's Theorem to the non-smooth case.

Theorem 1.1. Let $P$ and $Q$ be lattice polygons such that the normal fan of $Q$ coarsens that of $P$. Then the map $\mathfrak{s}$ is surjective.

Our proof originated in a discussion about normality of polytopes during a miniworkshop at Oberwolfach [HHM07.

The assumption on the normal fan is necessary. See Figure 1 for an example of two lattice polygons that do not satisfy the condition on the normal fan. The point $(0,0) \in P+Q$ cannot be written as a sum of a lattice point in $P$ and one in $Q$.

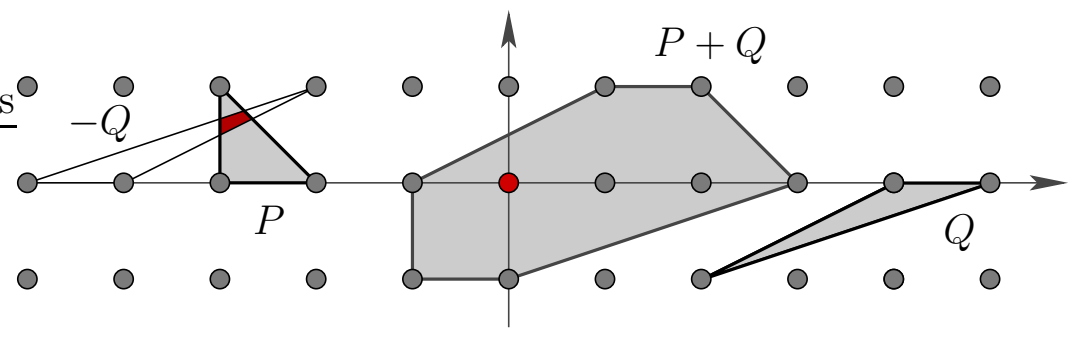

Figure 1: Theorem 1.1 fails without the assumption on the normal fan.

Theorem 1.1] remains true if $Q$ is a segment. Embarrassingly, the conjecture is open even if $\operatorname{dim}(P)=3$ and $\operatorname{dim}(Q)=2$, or if $P=Q$ and $\operatorname{dim}(P)=3$. Observe that in dimension three and higher the smoothness hypothesis cannot be removed. For example, if $P=Q$ is the simplex of lattice volume two in $\mathbb{R}^{3}$ having the four vertices as its only lattice points, then the centroid of $P+P$ is a lattice point but it is not in the image of the map $\mathfrak{s}$. 


\section{Lattice point free intersections are 4-gons}

As an intermediate step we prove the following curious result.

Proposition 2.1. Let $P$ and $Q$ be lattice polygons and let $Z=P \cap Q$. If $Z$ is not empty but does not contain a lattice point, then $Z$ is a 4-gon with two opposite edges coming from $P$ and the other two coming from $Q$.

Examples of the stated 4-gons appear in Figures 1 and 2

Proof. Let $Z:=P \cap Q$. If some vertex of $Z$ is a vertex of $P$ or of $Q$, then it is a lattice point in $Z$. So, let us assume that all vertices of $Z$ arise from an edge of $P$ and an edge of $Q$ intersecting in their relative interiors. This implies that $Z$ is two-dimensional, and that edges of $Z$ are alternatingly edges of $P$ and of $Q$. In particular, $Z$ has an even number $n \geq 4$ of edges.

We prove the theorem by contradiction. For this, assume $n \geq 6$ and let $\mathcal{L}(P)$ denote the set of lattice points in $P$ that are not vertices of $P$. We may assume that $P$ minimizes $|\mathcal{L}(P)|$ among the polygons for which $Z=P \cap Q$ has more than four edges and contains no lattice point.

If $\mathcal{L}(P)=\emptyset$, then $P$ is contained in a (closed) strip $R$ of lattice width one. The interior of $R$ intersects precisely two edges of $Q$, since the strip contains no lattice point in its interior. Those two are the only edges of $Q$ that can contribute to edges of $Z$. Hence, $Z$ is in fact a 4 -gon. See Figure 2

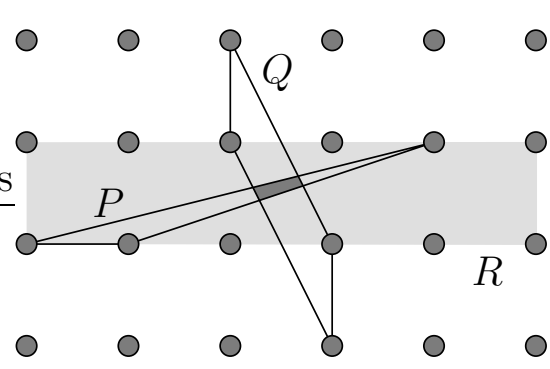

Figure 2: All lattice points of $P$ are vertices

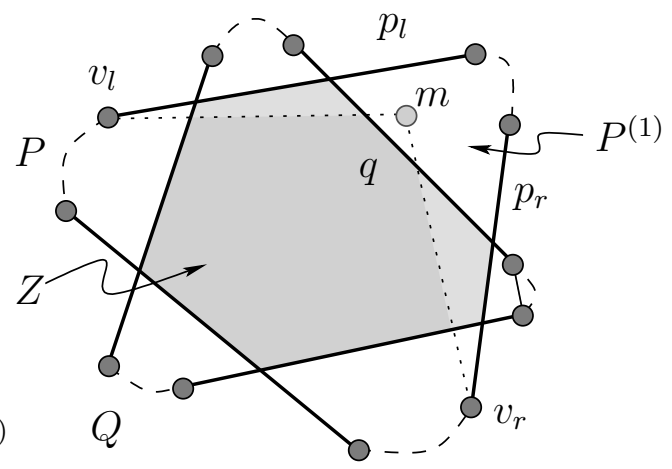

Figure 3: $m \in P \cap \mathbb{Z}^{2}$ is not a vertex of $P$

Now assume $|\mathcal{L}(P)|>0$. We will construct a subpolytope $P^{\prime} \subset P$ with $\left|\mathcal{L}\left(P^{\prime}\right)\right|<$ $|\mathcal{L}(P)|$ and such that the intersection $Z^{\prime}=P^{\prime} \cap Q$ has the same number of edges as $Z$, a contradiction.

For this, let $m \in \mathcal{L}(P)$. By assumption, $m \notin Z$. Hence, there is an edge $q$ of $Z$ with $m$ in its outer half-space $H^{+}$. As $q$ comes from an edge of $Q$, both $Q$ and $Z$ are contained in the closed half-space $H^{-}$. See Figure 3 .

Let $p_{l}, p_{r}$ be the edges of $Z$ adjacent to $q$. Then $p_{l}$ (respectively, $p_{r}$ ) is part of an edge $\overline{p_{l}}$ (respectively, $\overline{p_{r}}$ ) of $P$. Let $v_{l}$ (respectively, $v_{r}$ ) be the vertex of $\overline{p_{l}}$ (respectively, $\overline{p_{r}}$ ) contained in $H^{-}$. Since $Z$ is not a 4 -gon we have $v_{l} \neq v_{r}$. 
We define $P^{\prime}$ as the convex hull of $m$ and all vertices of $P$ that are contained in $H^{-}$. By construction, $Z^{\prime}:=P^{\prime} \cap Q$ is a 2-dimensional polygon with the same number of vertices as $Z$. As $m$ is a vertex of $P^{\prime}, \mathcal{L}\left(P^{\prime}\right) \subsetneq \mathcal{L}(P)$

\section{Proof of Theorem 1.1}

We first translate Theorem 1.1 into a statement that does not involve the map $\mathfrak{s}$ anymore. The following necessary and sufficient condition for $\mathfrak{s}$ to be surjective is due to Benjamin Howard How07.

Lemma 3.1. $\mathfrak{s}$ is surjective if and only if for all $z \in \mathbb{Z}^{2}$

$$
P \cap(z-Q) \neq \emptyset \quad \Longleftrightarrow \quad(P \cap(z-Q)) \cap \mathbb{Z}^{2} \neq \emptyset
$$

Proof. The left-hand side is equivalent to $z \in P+Q$. The right-hand side to $z \in(P \cap$ $\left.\mathbb{Z}^{2}\right)+\left(Q \cap \mathbb{Z}^{2}\right)$.

For example, in Figure 1 the point $(0,0)$ is not in the image of $\mathfrak{s}$ because the intersection of $P$ and $-Q$ does not contain a lattice point. Using this lemma, Theorem 1.1 is equivalent to the following.

Theorem 3.2. Let $P$ and $Q$ be lattice polygons. If $P \cap Q \neq \emptyset$ and the normal fan of $-Q$ coarsens that of $P$, then $P \cap Q$ contains a lattice point.

Proof. Let $Z=P \cap Q$ and suppose that $Z \cap \mathbb{Z}^{2}$ was empty. Then, by Proposition 2.1. $Z$ is a 4-gon with two opposite edges coming from $P$ and the other two coming from $Q$.

Let $e_{1}$ and $e_{2}$ be the edges of $Z$ originating from $P$ and $f_{1}, f_{2}$ those from $Q$. Let $\bar{f}_{1}$ and $\bar{f}_{2}$ be the edges of $P$ with exterior normals opposite to those of $f_{1}$ and $f_{2}$, respectively. They exist by the condition on the normal fans.

By construction, $\bar{f}_{2}, f_{1}, f_{2}$ and $\bar{f}_{1}$ are all contained, and appear in this order, in the (possibly degenerate) wedge defined by the lines supporting $e_{1}$ and $e_{2}$. See Figure 4 .

In particular, at least one of the $\bar{f}_{i}$ is shorter than or equal to its corresponding $f_{i}$. But since $\bar{f}_{i}$ is a lattice segment, and since $f_{i}$ is parallel to it, contained in a lattice line, and equal to or longer than it, $f_{i}$ must contain a lattice point. This contradiction finishes the proof.

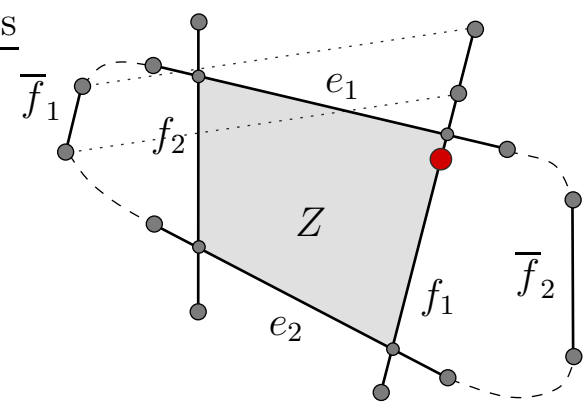

Figure 4: A lattice point on $f_{1}$. 
Note: Lev Borisov (unpublished) as well as Daiki Kondo and Shoetsu Ogata (unpublished) alerted us that they also proved Theorem 1.1 independently. Borisov's proof proceeds along the same lines as ours. His treatment of Proposition 2.1 is different. Kondo/Ogata generalize Fakhruddin's argument. Vladimir Danilov and Gleb Koshevoy discuss cases in which $\mathfrak{s}$ is surjective [DK04].

\section{References}

[DK04] Vladimir I. Danilov, Gleb A. Koshevoy. Discrete convexity and unimodularity. I, Adv. Math., 189(2): 301-324, 2004.

[Fak02] Najmuddin Fakhruddin. Multiplication maps of linear systems on smooth projective toric surfaces. Preprint, math.AG/0208178, 2002.

[HHM07] Christian Haase, Takayuki Hibi, and Diane MacLagan, editors. Mini-Workshop: Projective normality of smooth toric varieties, volume 39 of Oberwolfach report, 2007.

[How07] Benjamin J. Howard. Matroids and geometric invariant theory of torus actions on flag spaces. J. Algebra, 312(1):527-541, 2007.

[Oga06] Shoetsu Ogata. Multiplication maps of complete linear systems on projective toric surfaces. Interdiscip. Inf. Sci., 12(2):93-107, 2006. 